\title{
Decreased biomass recalcitrance effect and enhanced hydrolysis using ionic liquids: toward improvements in isofraxidin extraction
}

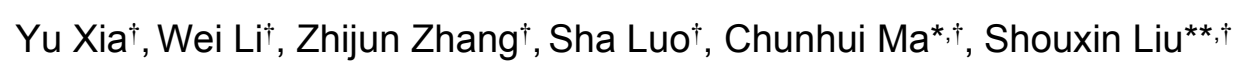

† Key Laboratory of Bio-based Material Science and Technology, Ministry of Education, College of Material Science and Engineering, Northeast

Forestry University, Harbin 150040, China

\footnotetext{
Corresponding Author

*Tel.: +86-451-82191204, E-mail: mchmchmchmch@163.com (Chunhui Ma);

Tel.: +86-451-82191502, E-mail: liushouxin@126.com (Shouxin Liu).
} 


\section{Supplementary Information}

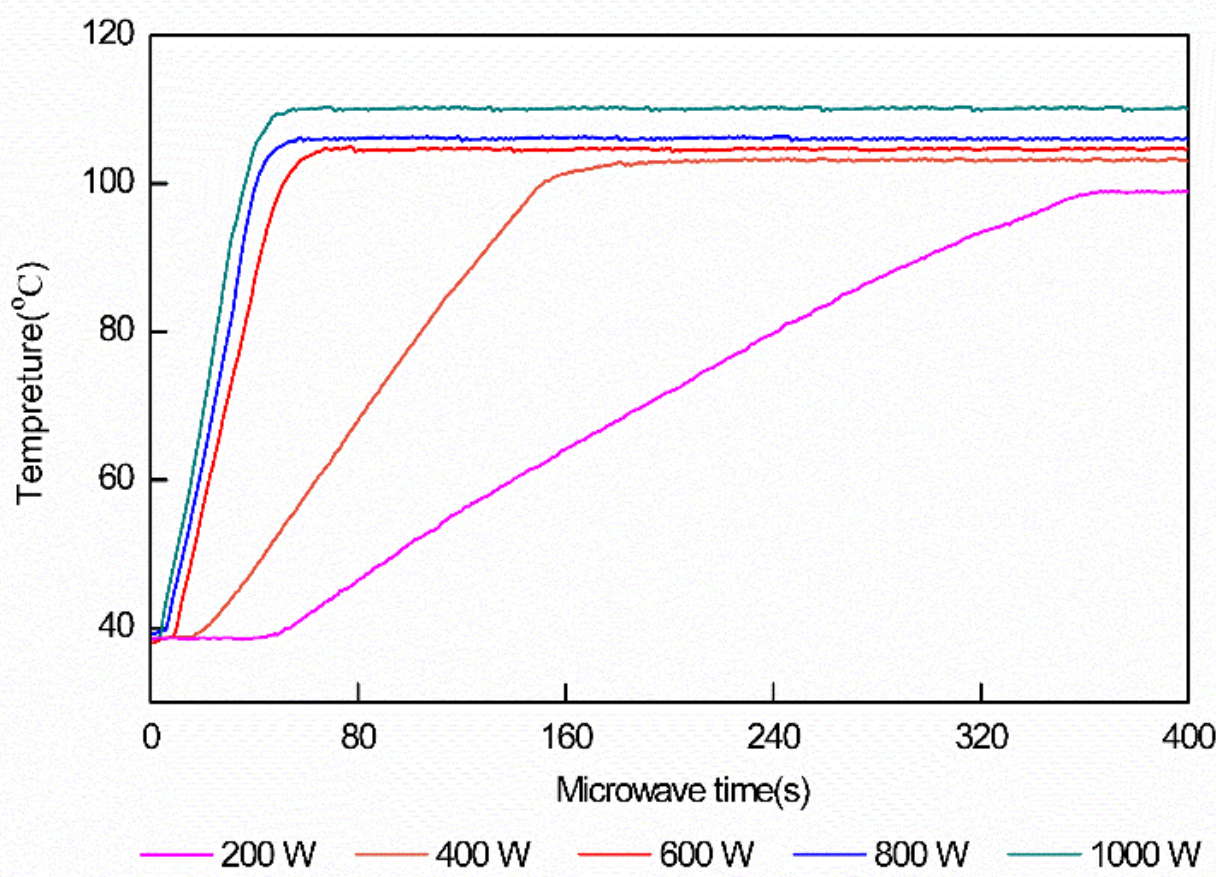

Figure S1 Heating curves of different power 
Table S1 Physical properties and absorption/desorption characteristics of the test macroporous resins

\begin{tabular}{|c|c|c|c|c|c|c|c|}
\hline Resin & $\begin{array}{c}\text { Surface } \\
\text { area }\left(\mathrm{m}^{2} / \mathrm{g}\right)\end{array}$ & $\begin{array}{l}\text { Average pore } \\
\text { diameter }(\AA)\end{array}$ & $\begin{array}{c}\text { Particle } \\
\text { diameter }(\mathrm{mm})\end{array}$ & Polarity & $\begin{array}{c}\text { Moisture } \\
\text { content (\%) }\end{array}$ & $\begin{array}{c}\text { Adsorption } \\
\text { capacity }(\mathrm{mg} / \mathrm{g})\end{array}$ & $\begin{array}{c}\text { Desorption } \\
\text { ratio }(\%)\end{array}$ \\
\hline HPD100 & $650-700$ & $85-90$ & $0.30-1.20$ & Non-polar & 65.00 & $4.33 \pm 0.23$ & $44.03 \pm 2.12$ \\
\hline HPD300 & $800-870$ & $50-55$ & $0.30-1.20$ & Non-polar & 75.52 & $4.67 \pm 0.24$ & $57.15 \pm 2.90$ \\
\hline HPD400 & $500-550$ & $75-80$ & $0.30-1.20$ & Polar & 68.93 & $4.33 \pm 0.21$ & $43.39 \pm 2.23$ \\
\hline HPD600 & $550-600$ & 80 & $0.30-1.20$ & Polar & 69.32 & $3.44 \pm 0.19$ & $61.27 \pm 3.12$ \\
\hline HPD700 & $650-700$ & $85-90$ & $0.30-1.20$ & Middle-polar & 66.10 & $4.55 \pm 0.22$ & $41.86 \pm 2.23$ \\
\hline HPD750 & $650-700$ & $85-90$ & $0.30-1.20$ & Middle-polar & 57.58 & $4.43 \pm 0.19$ & $42.27 \pm 2.19$ \\
\hline HPD850 & $1100-1300$ & $85-95$ & $0.30-1.20$ & Middle-polar & 46.81 & $4.22 \pm 0.16$ & $56.74 \pm 2.56$ \\
\hline AB-8 & $480-520$ & $130-140$ & $0.30-1.25$ & Weak-polar & 65.00 & $4.23 \pm 0.16$ & $83.43 \pm 1.47$ \\
\hline
\end{tabular}

where mean \pm S.D., $n=3$. 
Table S2 Langmuir and Freundlich parameters of isofraxidin on AB-8 at $25^{\circ} \mathrm{C}, 30^{\circ} \mathrm{C}$ and $35^{\circ} \mathrm{C}$

\begin{tabular}{|c|c|c|c|c|c|c|}
\hline \multirow{2}{*}{ Temperature $\left({ }^{\circ} \mathrm{C}\right)$} & \multicolumn{3}{|c|}{ Langmuir equation } & \multicolumn{3}{|c|}{ Freundlich equation } \\
\hline & Qmax & $K_{\mathrm{L}}$ & $R^{2}$ & $K_{\mathrm{F}}$ & $n$ & $R^{2}$ \\
\hline 25 & 6.33 & 434.78 & 0.9972 & 35.1965 & 1.7015 & 0.9622 \\
\hline 30 & 5.42 & 289.02 & 0.9857 & 38.9404 & 1.4943 & 0.9903 \\
\hline 35 & 4.79 & 208.33 & 0.9821 & 33.7054 & 1.4347 & 0.9942 \\
\hline
\end{tabular}


Table S3 Effects of different ethanol-water solution as desorption solutions on desorption properties of AB-8 for isofraxidin

\begin{tabular}{|c|c|c|c|c|c|c|c|}
\hline Ethanol-water solution (v/v) & $30: 70$ & $40: 60$ & $50: 50$ & $60: 40$ & $70: 30$ & $80: 20$ & $90: 10$ \\
\hline Content of dried elutes (mg) & $846 \pm 142$ & $873 \pm 110$ & $946 \pm 194$ & $1024 \pm 188$ & $1056 \pm 124$ & $1383 \pm 232$ & $1852 \pm 265$ \\
\hline Content of isofraxidin (mg) & $15.54 \pm 1.58$ & $21.44 \pm 2.73$ & $27.18 \pm 2.12$ & $31.62 \pm 2.22$ & $33.51 \pm 2.38$ & $36.82 \pm 2.42$ & $40.98 \pm 2.42$ \\
\hline Purity of isofraxidin (\%) & 1.84 & 2.46 & 2.87 & 3.09 & 3.17 & 2.66 & 2.21 \\
\hline
\end{tabular}

where mean \pm S.D., $n=3$. 


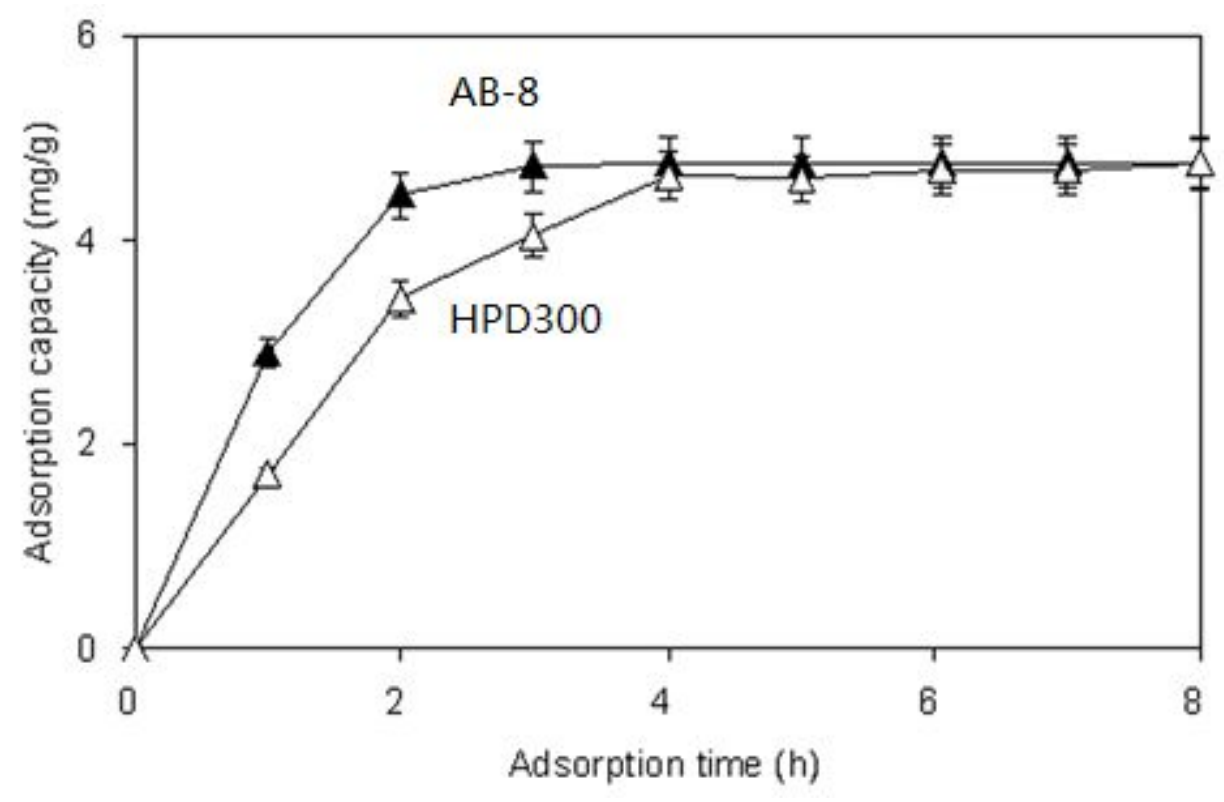

Figure S2 Adsorption kinetics curves for isofraxidin on AB-8 and HPD300 


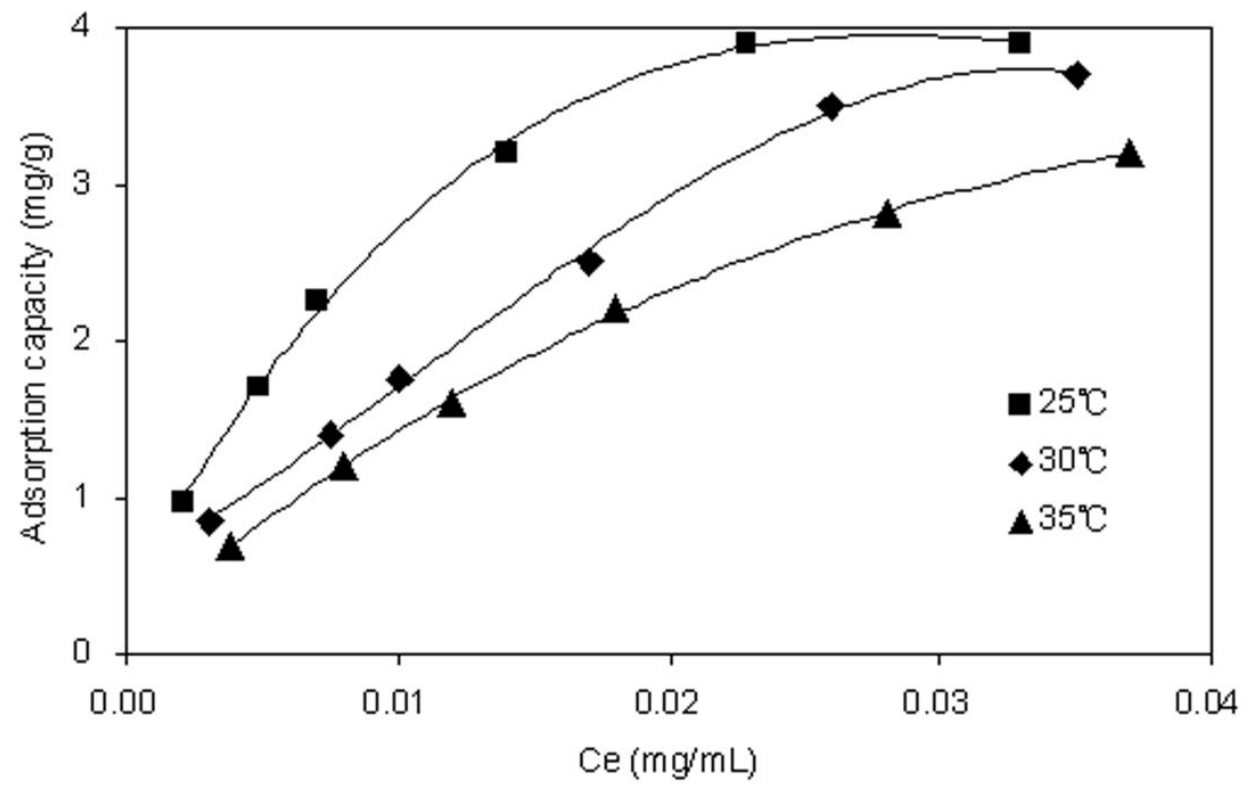

Figure S3 Adsorption isotherm at 25,30 and $35{ }^{\circ} \mathrm{C}$ for isofraxidin on $\mathrm{AB}-8$ 


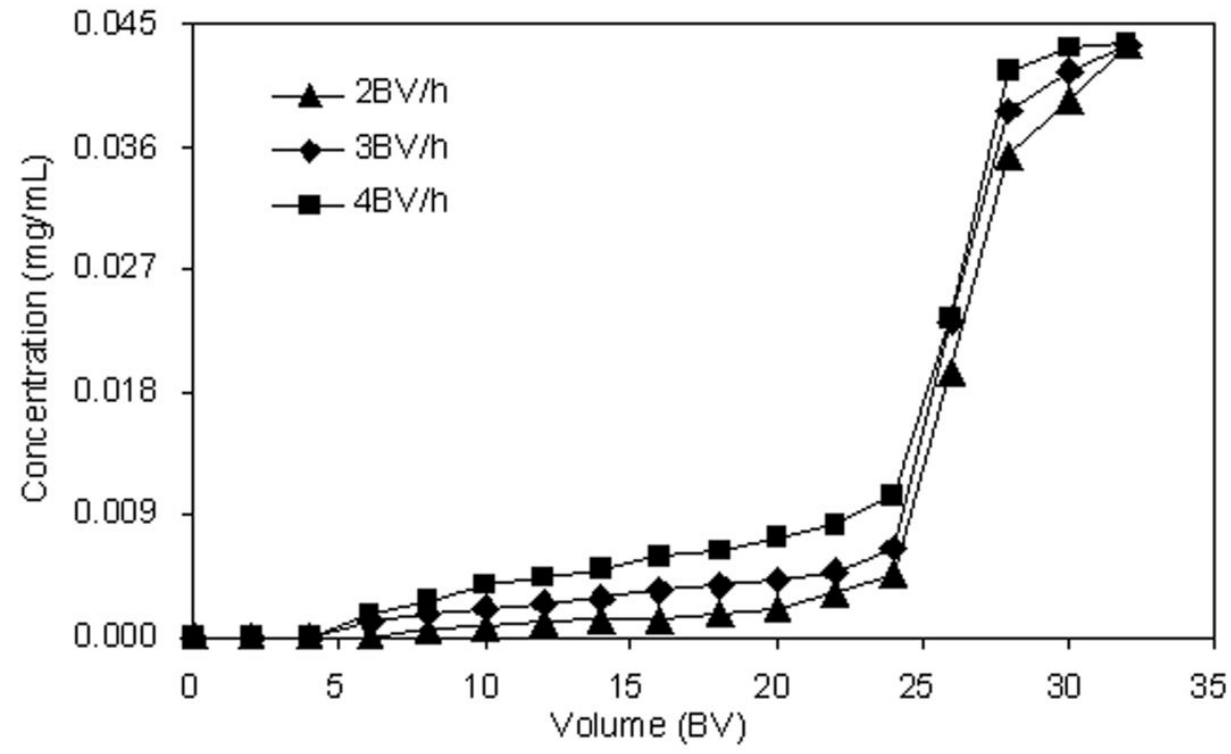

Figure S4 Dynamic leakage curves of isofraxidin on AB-8 


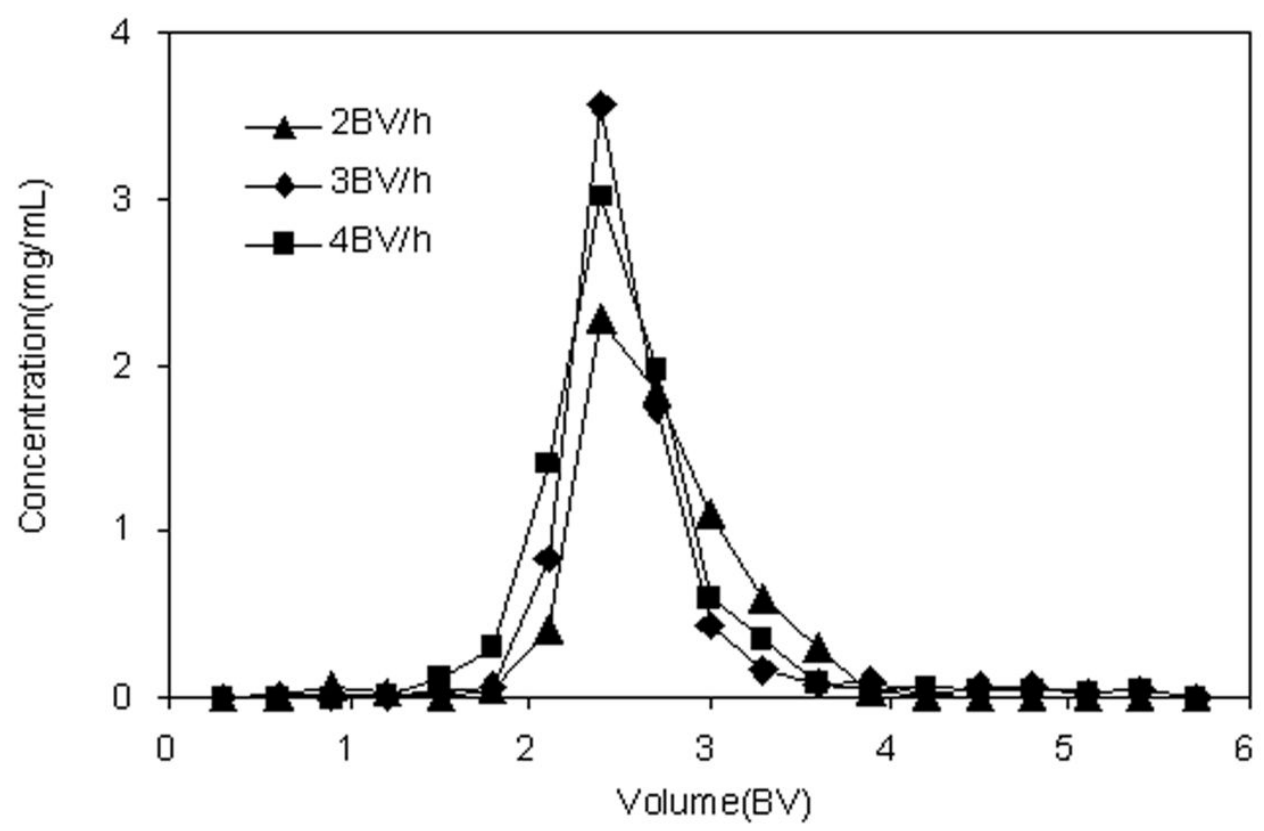

Figure S5 Dynamic elution curves of isofraxidin on AB-8 